\title{
Investigation on multi-dimensional energy saving system
}

\author{
Fuzhong Nian ${ }^{\mathrm{a}}$, Baochao Wang ${ }^{\mathrm{b}}$ \\ School of Computer \& Communication, Lanzhou University of \\ Technology, Lanzhou, 730050,China \\ agdnfz@lut.cn,bforever-wbc@163.com
}

\begin{abstract}
With the development of Internet of things technology, the energy saving system has been investigated extensively. However, So far there is little research on multi-dimensional dynamic smart system. In this paper, a multi-dimensional smart control system was designed and developed based on internet of things. To realize the saving energy, almost all of energy costing devices were controlled by computer in a home. For example, air-condition, light, ventilator and etc. The system includes software and hardware, the software is made up of server and client, and the hardware includes computer, single chip, all kinds of sensors, relative devices, and etc. The experiment shows that our system is effective and the energy costing was reduced sharply too.
\end{abstract}

Keywords: The Internet of Things, MCU, Sensor, Data Base, Energy Saving

\section{Instruction}

The energy saving has been a hot topic[1,2]. And, at same time, it is very important that the comfort would not be reduced. Thus, the energy saving research must ensure the comfort of users. In this paper, all kind of devices were integrated into a special system. And, the control decision is made according to the reflecting of the users in the home.

For a good system, the compatibility and expansibility is very important. In this system computer and cell phone are adopted as a control terminal network. On the computer system, a large number of laboratory actual data is collected as the foundation, designed and implemented the database to generate dynamic 
control commands. As a mobile terminal, mobile phone's flexibility and convenience is unparalleled. As a template system, more functionality sensors and more terminal equipments can be extended, in order to realize more complex functions[3].

\section{Design of the Hardware}

MCU control circuit board and room model are included in the hardware design part. We think an unified specification and standard is not formed in the smart home field resulting in the cost and use problems that make it unable to popularize at the moment. So we should put emphasis on usability and scalability of the system. On the hardware side, lighting control, air-conditioning control, humidifier and curtain control are integrated. On the basis, in the development process of the system the extended interfaces are reserved, so it's easy to let terminal equipment into the net.

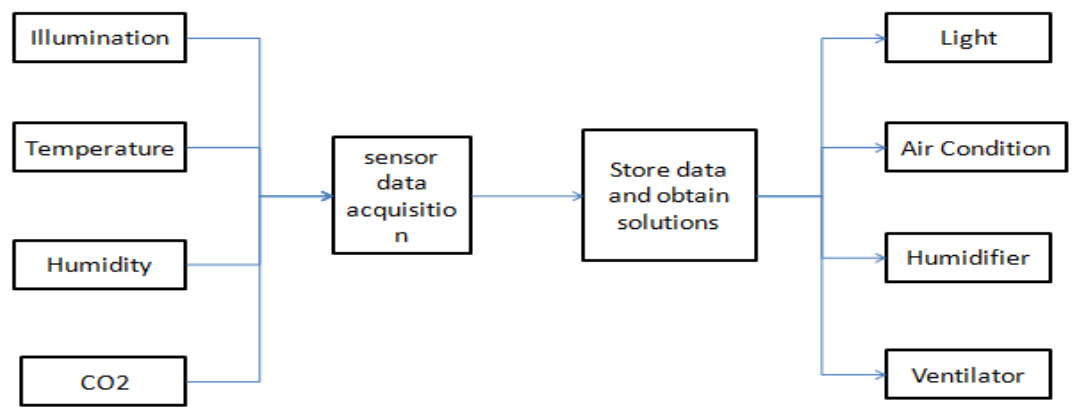

Fig. 1 Design of the hardware

MCU module, data acquisition and control parts used the STC89C51 control chips. STC89C51 has low power consumption, high performance CMOS 8 bit microcontroller, to make them available to provide flexible solution for many embedded control system. At present, most of the smart home control system is based on the single-chip microcomputer control system on the market.

Temperature and humidity sensor, photosensitive sensor, CO2 concentration sensor is included, in order to collect temperature, humidity, illumination, CO2 concentration respectively. These data will be stored in the database. Through 
our complaints based feedback mechanism, and the current environment parameters to generate scientific control commands to control household devices.

Temperature and humidity sensor DHT11 is used to realize temperature and humidity acquisition. DHT11 is a new kind of intelligent temperature and humidity sensor. Analog signal will be translated into digital signal, then through the single-chip computer to realize the data acquisition.

Photosensitive sensors are adopted in the light collection module. In the automatic control, photosensitive resistance sensor has a wide range of applications. In this system illumination is an important parameter that decide when to open and close the curtains.

$\mathrm{CO} 2$ is another important influencing factor that influences people's life comfortable degree, also the important factors influencing the opening windows, switching fans. A too high CO2 degree will cause a decline in people's comfort level, concentration and a series of negative effects. As a part of the system, it is also very necessary to integrate CO2 concentration sensor[4].

PM2.5 has been a hot topic in the field of smart home. Ultrafine particle refers to the aerodynamic diameter less than or equal to 2.5 micron particles in the environment. It can be suspended in the air for a long time. PM2.5 damage people's organs, and has a strong carcinogenic. Because of this, PM2.5 is considered in our system. Because of the PM2.5 module's price and the PM2.5 value was close as long as the location is not far. So we collected real-time PM2.5 information through network, and gathered into the database together, used for generating commands.

\section{Design of the System Software}

\subsection{PC user interface design.}

On the aspect of software design, PC and mobile terminal is adopted as double control interface. Mobile client can be used, also as a web browser, for remote view and control. 
The web client device control interface, the web client interface indoor environment parameters, the web client default control interface, the web client complaints feedback interface is included in the interaction interface. It is the decision maker, receiving sensor real-time environment parameters, processing users' feedback information. Then according to the combination of comfortable environment model and feedback control command, realize the intelligent control of the whole system.

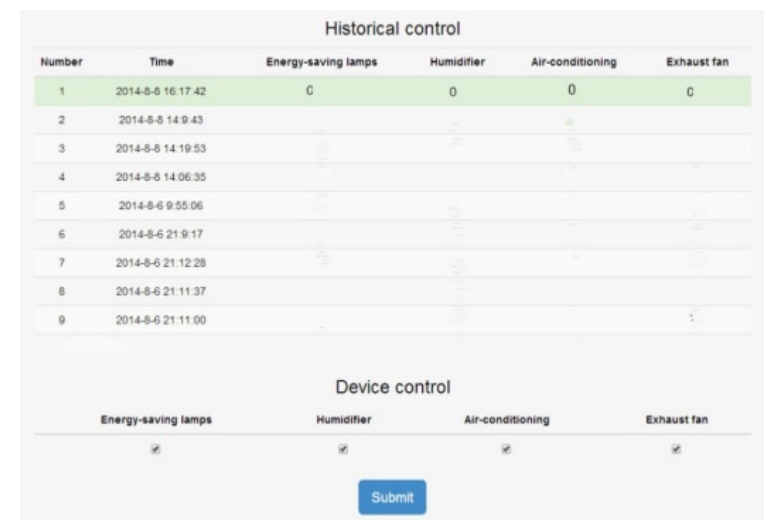

Fig. 2 Web client device control interface

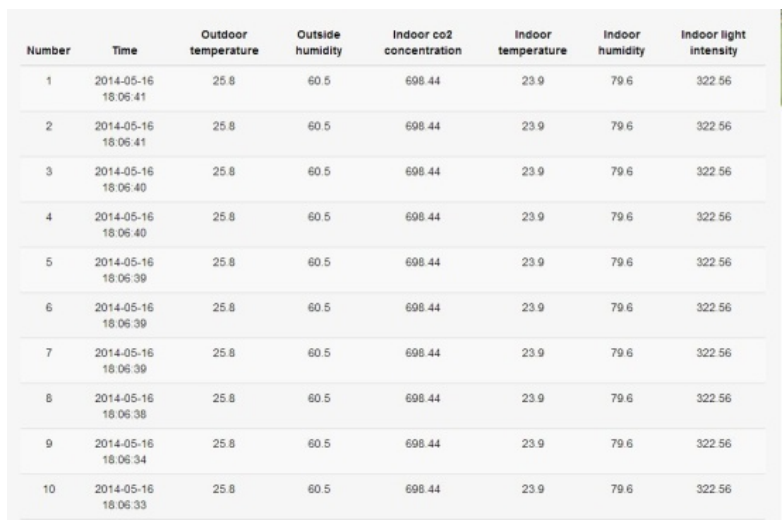

Fig. 3 Indoor environment parameters

Commands are send to control each household device through the device control interface. The system will give priority to user's direct control. The real-time state of household appliances and the detail of the environmental index are showed in the environment parameters interface, including indoor and 
outdoor temperature, CO2 concentration, humidity and illumination. These data can help users make reasonable judgment.

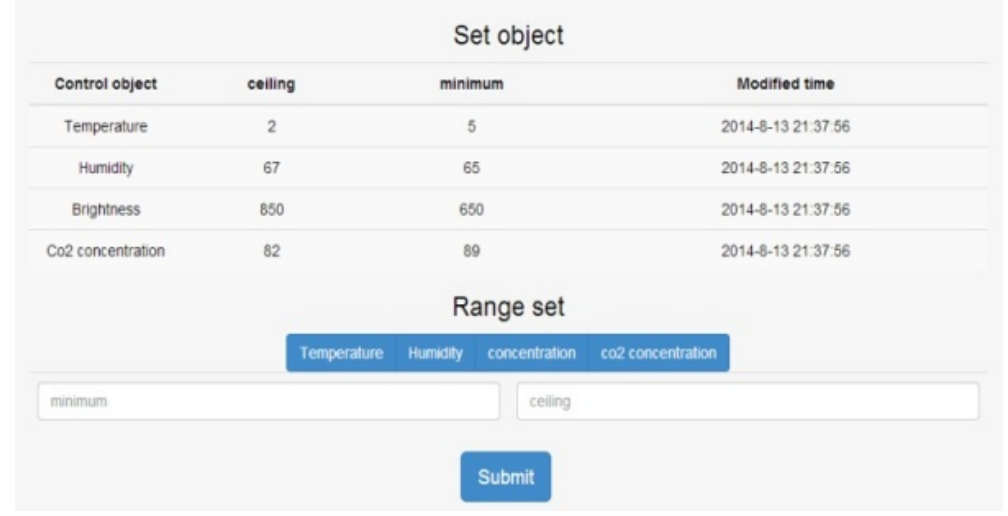

Fig. 4 Web client default regulation

\begin{tabular}{|c|c|c|c|c|c|c|c|c|c|}
\hline Number & Time & cold & Hot & Dry & Wet & Dark & Cool & Stuffy & User \\
\hline 1 & $2014-8-8$ 16-18-35 & 0 & 1 & 0 & 1 & 0 & 1 & 1 & davis \\
\hline 2 & $2014-8-711: 48-53$ & 1 & 1 & 1 & 1 & 1 & 1 & 1 & davis \\
\hline 3 & $2014-8-141-43-18$ & 0 & 0 & 0 & 1 & 0 & 1 & 0 & davis \\
\hline 4 & $2014-8-1410.48: 56$ & 0 & 1 & 0 & 0 & 0 & 0 & 0 & davis \\
\hline 5 & $2014-8-1019: 32: 57$ & 0 & 0 & 1 & 0 & 0 & 1 & 0 & davis \\
\hline 6 & $2014-8-1018: 29: 37$ & 1 & 1 & 0 & 1 & 0 & 1 & 1 & davis \\
\hline
\end{tabular}

Fig. 5 Web client complained feedback

A preset adjustment can be made according to your own personal preferences. Default values will be considered to generate the final order in the process of command algorithm. System uses a feedback mechanism based on complaints, user's feelings about the environment quality can be collected timely and accurately and the feelings of users' direct feedback will be considered by the decision maker.

\subsection{The design of mobile interfaces}

Mobile phone client is designed based on the android. Through the WIFI to connect to the home network. The whole system can be mastered by the users, 
and real-time control at any time any place. The display of real-time sensor data is included, in this way environment indicators can be monitored anywhere. Mobile client device control interface similar to the PC control interface. Terminal equipment can be directly controlled through the equipment control interface. Users' current feeling of environment can be collected by the users' complain feedback interface and it is the main basis that generated command algorithm. In order to make the system's functions more comprehensive, mobile client weather is gathered into our system, allow the users to know the weather and help the user make a proper decision.

\section{Experiments}

Energy saving is a prominent feature of the system. We have the facilities of the laboratory for the system to provide the full range of reliable data. Our system's purpose is to try to achieve energy-saving and not reduce the comfort level[5].

Based on the control data of Lanzhou laboratory as an object. On November 6 , temperature acquisition data is illustrated below.

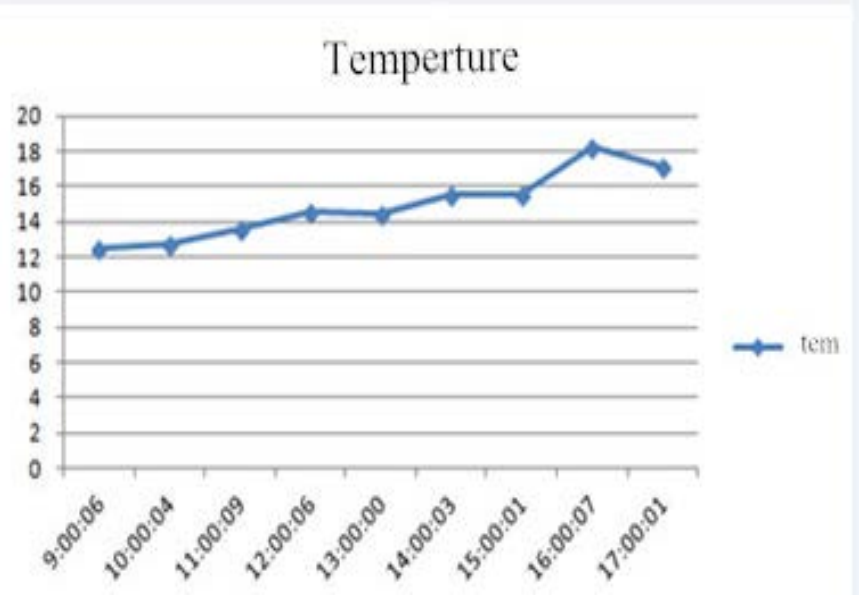

Fig. 6 Temperature data acquisition 


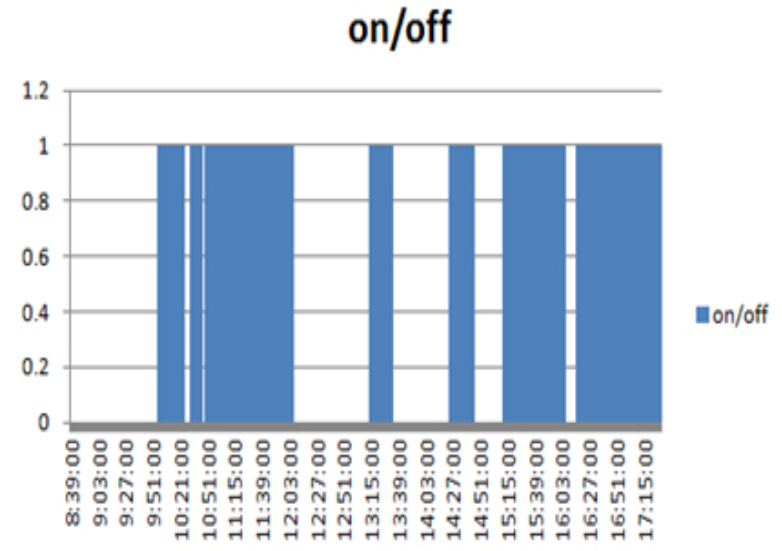

Fig. 7 Air conditioning switch state data

Our energy-saving idea has two aspects. First of all, if there is someone in the laboratory, a minimum value of time is taken to open air condition on an acceptable range of complaints. Secondly, there is a common phenomenon, if the set point of air condition is not 'proper', the air condition has to work constantly in order to make the temperature lower a little. If the temperature set point is changed lower in advance, although just a little change, can make air condition work at intervals. For an example, if the set point is set by $25^{\circ} \mathrm{C}$, the air condition has to work without a break to reach the point, if the set point is set by $24^{\circ} \mathrm{C}$, the air condition may work with a lot of break. Small temperature difference may means nothing to the users' feeling, but means everything to the equipment. We call it the 'boundary effect of building energy efficiency', this character of the equipments will be considered by our algorithm in the future development.

Under the algorithm based on the complain feedback, on the premise of meet the requirements of most people and automatically shut down electric equipment. Through this method, comfortable environment can be realized while achieve the goal of energy saving.

\section{Conclusions}

From the point of the users, in this paper, the multi-dimensional energy saving system was investigated. The software and the hardware were designed, 
and all the energy costing devices were integrated. The control decision is made according to the reflecting of the users. The experiments indicate that the system is effective.

\section{Acknowledgments}

This research is supported by the National Natural Science Foundation of China (No: 61263019), Program for International S\&T Cooperation Projects of Gansu province(No.144WCGA166) and the Doctoral Foundation of LUT.

\section{References}

[1] Fuzhong Nian, Qianchuan Zhao, Pinning synchronization with low energy cost, Communications in Nonlinear Science and Numerical Simulation,2014,19(4):930-940.

[2] Xiaojing Ye; Junwei Huang, "A framework for Cloud-based Smart Home," Computer Science and Network Technology (ICCSNT), 2011 International Conference on , vol.2, no., pp.894,897, 24-26 Dec. 2011

[3] Hsien-Tang Lin, "The development of control and energy usage information modules for smart homes," Control, Automation and Information Sciences (ICCAIS), 2012 International Conference on , vol., no., pp.236,240, 26-29 Nov. 2012

[4] Adipradhana, M.; Nugraha, IG.B.B.; Supangkat, S.H., "Intervention of non-inhabitant activities detection in smart home environment," ICT for Smart Society (ICISS), 2013 International Conference on , vol., no., pp.1,5, 13-14 June 2013

[5] Elma, O.; Selamogullari, U.S.; Uzunoglu, M.; Ugur, E., "Carbon emission savings with a renewable energy supplied smart home operation," Renewable Energy Research and Applications (ICRERA), 2013 International Conference on , vol., no., pp.1129,1132, 20-23 Oct. 2013

[6] Dae-Man Han; Jae-Hyun Lim, "Smart home energy management system using IEEE 802.15.4 and zigbee," Consumer Electronics, IEEE Transactions on , vol.56, no.3, pp.1403,1410, Aug. 2010 